\title{
DOR: QUINTO SINAL VITAL, UM DESAFIO PARA O CUIDAR EM ENFERMAGEM ${ }^{1}$ \\ PAIN: FIFTH VITAL SIGN, A CHALLENGE FOR THE CARE IN NURSING DOLOR: QUINTA SEÑAL VITAL, UN RETO PARA EL CUIDAR EN ENFERMERÍA
}

\author{
Rene Antonio Pedroso², Kátia Lilian Sedrez Celich ${ }^{3}$
}

1 Trabalho monográfico de conclusão do Curso de Enfermagem da Universidade Regional Integrada do Alto Uruguai e das Missões (URI), Campus de Erechim - RS, como requisito para a obtenção do título de Bacharel em Enfermagem.

2 Acadêmico do nono semestre do Curso de Graduação em Enfermagem da URI, Campus de Erechim - RS.

3 Mestre em Enfermagem pela Escola de Enfermagem da Universidade Federal do Rio Grande do Sul (UFRGS). Doutoranda em Gerontologia Biomédica na Pontifícia Universidade Católica do Rio Grande do Sul (PUCRS). Professora do Curso de Graduação em Enfermagem da URI, Campus de Erechim - RS. Membro do Núcleo de Estudos do Cuidado em Enfermagem (NECE) do Hospital de Clínicas de Porto Alegre (HCPA).

PALAVRAS-CHAVE: Dor. Enfermagem. Educação. Assistência centrada no paciente.

KEYWORDS: Pain. Nursing. Education. Patient-centered care.

PALABRAS CLAVE: Dolor. Enfermería. Educación. Atención dirigida al paciente.
RESUMO: A dor é considerada uma experiência subjetiva, que deve ser avaliada e descrita como o quinto sinal vital. No entanto, se observa que esta prática é pouco freqüente nos ambientes hospitalares. Este estudo busca identificar o conhecimento da equipe de enfermagem em relação à avaliação da dor, sensibilizando-a para instituí-la como quinto sinal vital, no cotidiano profissional. Caracteriza-se como uma pesquisa quanti-qualitativa, realizada no Centro de Terapia Intensiva, num hospital do Rio Grande do Sul. Os participantes foram quatorze membros da equipe de enfermagem. Os resultados apontaram que esta equipe tem noção de apenas uma escala para avaliação da dor e reconhecem alguns sinais de dor, mas não tem como prática sistemática entendê-la como quinto sinal vital. Assim, ao avaliar os outros sinais, não investiga a dor. Acredita-se que a educação possibilita o cuidado qualificado, em que as reais necessidades do cliente são atendidas.

ABSTRACT: Pain is considered to be a subjective experience, which should be evaluated and described as the fifth vital sign. However, this practice is not observed to be frequent inside hospitals. Therefore, this study aims to identify the knowledge of the nursing team regarding evaluation of pain; to capacitate the team to evaluate it and to propose it to be considered as a fifth vital sign, in the professional quotidian. This study is characterized as a quantitative and qualitative research, carried out in the Intensive Therapy Center of a hospital in Rio Grande do Sul. Fourteen employees from the nursing team took part in the research. The results pointed out that the nursing team knows about the existence of scales to evaluate pain, as well as to recognize signs of pain. But they do not, as a systematic practice, see it as the fifth vital sign. So, when evaluating the other signs, they do not investigate pain. It is believed that education makes qualified care possible, in which the real needs of the client are fulfilled.

RESUMEN: El dolor es considerado una experiencia subjetiva, que debe ser evaluada y descripta con la quinta señal vital. Sin embargo se observa que esta práctica es poco frecuente en los ambientes hospitalarios. Este estudio busca identificar el conocimiento del equipo de enfermería con relación a la evaluación del dolor, sensibilizándola esto equipo para evaluarlo y proponer que sea instituido como quinta señal vital, en el cotidiano profesional. Se caracteriza como una pesquisa cuantitativo-cualitativa, realizada en el Centro de Terapia Intensiva en un hospital en Rio Grande do Sul. Los participantes fueron 14 funcionarios del equipo de enfermería. Los resultados apuntaron que esto equipo de enfermería conoce las escalas para evaluación del dolor, y reconoce las señales de dolor, pero, no tiene como práctica sistemática entenderla como quinta señal vital. Así, al evaluar las otras señales no investiga el dolor. Se cree que la educación posibilita el cuidado cualificado, en que las reales necesidades del cliente son atendidas.
Endereço: Rene Antonio Pedroso

R. José Sponchiado, 550

99.700-000 - Bela Vista, Erechim, RS.

E-mail: renepedr@bol.com.br
Artigo original: Pesquisa Recebido em: 10 de outubro de 2005. Aprovação final: 02 de maio de 2006. 


\section{INTRODUÇÃO}

Desde os primórdios, o homem vem, progressivamente, procurando compreender as causas da dor com a finalidade de livrar-se dela. Todas as pessoas, de um modo geral, sabem o que é dor. Entretanto, geralmente, é difícil para alguém descrever a própria dor e impossível conhecer exatamente a experiência de dor de outra pessoa. Essa dificuldade decorre do fato de que a dor é uma experiência individual, com características próprias do organismo, associada a sua história passada, além do contexto no qual ela é percebida. ${ }^{1}$

Dor é definida pela Sociedade Internacional para o Estudo da Dor (IASP) como uma experiência sensitiva emocional desagradável relacionada à lesão tecidual ou descrita em tais termos. ${ }^{2}$ Trata-se de uma manifestação subjetiva, que envolve mecanismos físicos, psíquicos e culturais.

Desde janeiro de 2000, a Joint Comission on Accreditation on Heathcare Organizations (JCAHO) publicou norma que descreve a dor como quinto sinal vital. ${ }^{3}$ Portanto, ela deve ser sempre avaliada e registrada ao mesmo tempo em que são verificados os outros sinais vitais. Esse ato é necessário para que exista conhecimento da conduta tomada, sua razão e seus resultados. ${ }^{4}$ A queixa de dor deve ser sempre valorizada e respeitada, devido ao desconforto que manifesta.

Durante a hospitalização, geralmente, o cliente é submetido a procedimentos dolorosos, sobretudo nos setores de terapia intensiva. Embora a dor se faça tão presente nesse cotidiano e seus estudos tenham evoluído muito nos últimos anos, ainda existem lacunas no conhecimento sobre esse evento. Vale ressaltar que a equipe de enfermagem é quem efetivamente convive mais tempo com o cliente; dessa forma, se faz necessário que saiba conhecer os sinais de dor para assim buscar intervir corretamente no seu alívio.

Os instrumentos para mensurar a dor podem ser unidimensionais ou multidimensionais. Escalas unidimensionais avaliam somente uma das dimensões da experiência dolorosa e, dentre as mais usadas, destacam-se a Escala Visual Numérica (EVN), graduada de zero a dez, na qual zero significa ausência de dor e dez, a pior dor imaginável, e a Escala Visual Analógica (EVA), que é um instrumento simples, sensível e reprodutível, permitindo análise contínua da dor, que consiste em uma linha reta, não numerada, indicandose em uma extremidade a marcação de "ausência de dor' e na outra, "pior dor imaginável". Sem dúvida, ambas escalas têm a vantagem de facilitar o contato cuidador e cliente, ao compartilhar-se a intensidade da dor, e fornece ao cliente um instrumento para "se fazer entender". 5,6

Um artifício muito usado na mensuração da dor é tentar comparar a intensidade da experiência álgica e exemplos da vida diária do cliente, pois a sua familiaridade com essa realidade estabelece cumplicidade suficiente entre cuidador e cliente, fazendo com que este perceba o quanto tentamos entendê-los como indivíduo, e não como um dado padronizado. A partir daí, transpomos esta informação para as escalas unidimensionais, e obtemos uma graduação específica. ${ }^{5}$

Classicamente, consideram-se três dimensões de dor: a sensorial-discriminativa, a motivacional-afetiva e a cognitiva-avaliativa, avaliadas em escalas chamadas multidimensionais - Inventário para dor Mcgill, Wisconsin Brief Pain Questionnaire - mas que, em função de sua elevada complexidade e difícil aplicabilidade e reprodutibilidade, têm seu uso restrito para situações específicas. ${ }^{6}$

A experiência dolorosa é evento muito mais amplo, não se resumindo apenas à intensidade. As características da dor também devem ser avaliadas, incluindo o seu início, local, irradiação, periodicidade, tipo de dor, duração e fatores desencadeantes. É importante observar as reações comportamentais e fisiológicas da dor, tais como: expressão facial, inquietação, posicionamento protetor, insônia, ansiedade, irritabilidade, sudorese, palidez, taquicardia, taquipnéia, hipertensão, entre outros. ${ }^{5}$

Estas considerações permitem entender que se faz necessário que a equipe de enfermagem esteja ciente de sua responsabilidade frente ao cliente com dor, pois se essa conseguir perceber seu papel de cuidador, poderá intervir de maneira positiva, respeitando o ser e contribuindo para a realização de um cuidado humanizado.

Logo, o presente trabalho tem como objetivo identificar o conhecimento da equipe de enfermagem em relação à avaliação da dor, sensibilizando essa equipe para instituí-la como quinto sinal vital no cotidiano profissional.

\section{CAMINHO METODOLÓGICO}

A presente pesquisa foi de natureza quantitativo-qualitativa. $\mathrm{O}$ estudo quantitativo se limita à estatística descritiva. Foi realizada no Centro de Tratamento Intensivo (CTI) de um hospital geral filantrópico, localizado em uma cidade do norte do Estado do Rio Grande do Sul. A referida instituição possui cento e 
dezoito leitos, destinados à atenção múltipla à saúde, apoiando o ensino e a pesquisa. A equipe de enfermagem do CTI dessa instituição constitui-se por dezesseis cuidadores, sendo treze técnicos de enfermagem e três enfermeiros, distribuídos em três turnos de trabalho: manhã, tarde e noite.

Essa escolha fundamenta-se pelo fato de ser o local onde se realizam estágios supervisionados I e II e voluntários, onde se percebeu a importância de se realizar uma reflexão com a equipe de enfermagem sobre a avaliação da dor, sendo que esta é considerada, internacionalmente, o quinto sinal vital.

Com a finalidade de deixar a equipe de enfermagem ciente dos propósitos e da metodologia da pesquisa, foram explicados os objetivos almejados com a referida temática para a equipe de cada turno de trabalho. Os participantes, de acordo com seu interesse, tiveram autonomia de escolher participar ou não da pesquisa, isto é, foram selecionados de maneira não intencional. Os participantes do estudo foram quatorze membros da equipe de enfermagem da referida CTI. Em atenção à resolução 196/96 do Conselho Nacional de Saúde, que legisla sobre diretrizes e normas regulamentadoras de pesquisas envolvendo seres humanos, ${ }^{7}$ as participantes que concordaram fazer parte da pesquisa assinaram um termo de consentimento denominado Termo de Consentimento Livre e Esclarecido. Nesse termo, ficou assegurado o sigilo de sua identidade.

Solicitou-se autorização para a realização do estudo à Coordenação de Enfermagem, ao Comitê de Ética do Hospital e ao Comitê de Ética em Pesquisa da Universidade, a fim de se obter permissão para realizar a pesquisa na referida instituição.
A coleta dos dados aconteceu através de um questionário semi-estruturado com perguntas fechadas e abertas, com o propósito inicial de identificar o conhecimento dos participantes quanto à avaliação da dor. Após, realizou-se quatro palestras com cada equipe de enfermagem, em seu devido turno de trabalho, contemplando, assim, todo o grupo do CTI, com a finalidade de sensibilizar a equipe para a avaliação da dor, a fim de entendê-la como quinto sinal vital. Nas palestras, a temática foi abordada de maneira clara e objetiva, proporcionando um entendimento da necessidade da avaliação da dor e de como avaliá-la, permitindo aos participantes esclarecerem qualquer dúvida, elucidando que estaríamos a disposição para qualquer esclarecimento.

Os dados coletados foram organizados em tabelas e procedeu-se à análise descritiva. Nessa etapa, buscou-se estabelecer uma compreensão dos dados coletados, confirmando ou não os pressupostos da pesquisa e/ou responder às questões formuladas, bem como ampliar o conhecimento sobre o assunto.

\section{APRESENTAÇÃO E DISCUSSÃO DOS DADOS}

Apresentamos a seguir as informações colhidas em relação ao conhecimento da equipe de enfermagem sobre as formas utilizadas para se avaliar a dor do cliente hospitalizado na CTI.

Os participantes da pesquisa entendem que a dor pode ser avaliada pelos sinais de dor, seguidos da queixa verbal do cliente, da observação dos sinais vitais e da avaliação da escala de dor - Escala analógica visual (VAS), como mostra a Tabela 1.

Tabela 1 - Análise do conhecimento sobre a avaliação da dor na UTI. Erechim - RS, 2005.

\begin{tabular}{lcl}
\hline Conhecimento sobre avaliação da dor & Respostas obtidas* & $\mathbf{\%}$ \\
\hline Sinais de dor & 11 & 45,83 \\
Queixa verbal & 07 & 29,17 \\
Sinais vitais & 05 & 20,83 \\
Escala de dor (VAS) & 01 & 4,17 \\
\hline Total & $\mathbf{2 4}$ & $\mathbf{1 0 0}$
\end{tabular}

* Um mesmo participante da pesquisa respondeu mais de um item, o que fez aparecer vinte e quatro respostas.

Examinando-se as respostas, observa-se que existe um certo entendimento de como pode ser avaliada a dor, uma vez que $45,83 \%$ reconhecem os sinais de dor através da expressão facial, inquietação, sudorese, palidez, posicionamento protetor, entre outros. É importante observar que a valorização da queixa verbal de dor é o dado que se segue, apresentando 29,17\% de entendimento. A alteração dos si- 
nais vitais também é reconhecida por alguns dos participantes $(20,83 \%)$, como sendo uma possibilidade de constatação de dor. A escala de avaliação da dor aparece apenas como a escala analógica visual (VAS), o que permite inferir ser necessário ampliar o conhecimento a outras escalas. É muito importante identificar as características que expressam a dor, pois, em alguns casos, existem clientes que apresentam diminuição do nível de consciência, não podendo manifestar verbalmente a sua experiência dolorosa. Notase que os sinais de dor e as alterações dos sinais vitais são bastante observadas pela enfermagem, porque são indicadores da presença de dor.

Existem algumas características definidoras da existência da dor, dentre as quais podemos citar o relato verbal ou codificado; gestos protetores; expressão facial; distúrbio do sono; respostas autonômicas (diaforese, alterações dos sinais vitais, dilatação pupilar); comportamento expressivo (gemência, agitação, choro, irritabilidade); mudanças na alimentação; aparência abatida; interação reduzida com o ambiente. ${ }^{8}$

O papel principal do cuidador é ajudar o cliente a transmitir a sensação dolorosa de modo mais eficaz. As escalas de dor são úteis não apenas na determinação da intensidade da dor, mas também na avaliação de alterações da intensidade após a terapia ou quando os sintomas se agravam. ${ }^{9}$

Estudo realizado com enfermeiras de CTI pediátrica indicam que sensibilizar-se é primordial para que a enfermeira comprometa-se com o alívio da dor. Todas as experiências vividas por ela e os sentimentos associados não terão representação, se não houver sensibilização por parte do profissional que vivenciou experiência. ${ }^{10}$

Tabela 2 - Verificação dos sinais vitais e a avaliação da dor. Erechim - RS, 2005.

\begin{tabular}{lcl}
\hline Verificação dos sinais vitais e avaliação da dor & Número de informantes & $\mathbf{\%}$ \\
\hline Às vezes & 12 & 85,71 \\
Sempre & 02 & 14,29 \\
\hline Total & $\mathbf{1 4}$ & $\mathbf{1 0 0}$ \\
\hline
\end{tabular}

De acordo com a tabela acima, observa-se que, dentre os participantes da pesquisa, doze destes, às vezes, perguntam se o cliente está com dor ao verificar os sinais vitais, enquanto que apenas dois participantes sempre fazem essa pergunta. Esses números demonstram que a dor é pouco avaliada quando se verificam os demais sinais vitais, fato que pode ser explicado pelo grande número de cuidadores terem respondido investigar a dor somente às vezes. Assim, é mister que a avaliação da dor seja instituída como quinto sinal vital, já que ela o representa. Acredita-se que a educação em saúde pode assegurar uma prática mais qualificada; portanto essa se caracteriza como um desafio no cuidar em enfermagem.

Avaliar a dor como quinto sinal vital é uma maneira de melhorar a qualidade de vida do cliente, pois a dor é um dos mais freqüentes sintomas relatados por estes. ${ }^{5} \mathrm{~A}$ avaliação da dor, rotineiramente, possibilita planejar a medicação, de acordo com as necessidades pessoais e permite verificar a eficácia dos tratamentos de modo confiável. ${ }^{11} \mathrm{O}$ ambiente do CTI com todos os seus recursos tecnológicos e científicos que poderiam assegurar um cuidado eficaz ao cliente, em relação à avaliação da dor cotidiana, ainda é precário.

A dor é considerada um sinal vital tão importante quanto os outros e deve sempre ser avaliada num ambiente clínico, para se empreender um tratamento ou conduta terapêutica. A eficácia do tratamento e o seu seguimento dependem de uma avaliação e mensuração da dor confiável e válida. ${ }^{12}$

Observou-se, conforme tabela acima, que oito cuidadores nem sempre acreditam nas palavras do indivíduo que refere dor. Entretanto, seis cuidadores acreditam no relato do cliente da existência da dor.

Os cuidadores que assinalaram nem sempre acreditar no relato do cliente entendem que alguns querem chamar atenção por estarem apresentando ansiedade, insegurança, distância da família e carência afetiva. Eles percebem ser importante conhecer o cliente e avaliar até que ponto vai a dor; se esta provém de determinadas patologias ou vontade de tomar medicação.

Os membros da equipe de enfermagem que acreditam no relato do cliente, quando este refere estar com dor, justificam sua resposta por entenderem ser possí- 
Tabela 3 - Credibilidade na expressão verbal do cliente. Erechim - RS, 2005.

\begin{tabular}{lcc}
\hline Credibilidade na informação verbal do cliente & Número de informantes & $\mathbf{\%}$ \\
\hline Nem sempre & 08 & 57,14 \\
Sempre & 06 & 42,86 \\
\hline Total & $\mathbf{1 4}$ & $\mathbf{1 0 0}$ \\
\hline
\end{tabular}

vel que o cliente esteja sentindo dor; por isso, investigam a sua origem; associam a dor de acordo com certas patologias. Porém, afirmam não ser possível medir a dor, portanto, devem confiar no relato do cliente.

Analisando as respostas acima, percebe-se que a maioria dos cuidadores nem sempre acredita na dor do cliente, porém dor é tudo o que o indivíduo diz ser dor e existe sempre que o mesmo assim afirmar. ${ }^{3} \mathrm{Na}$ própria definição de dor, proposta pela Associação Internacional para Estudo da Dor, fica evidente que a dor é uma experiência subjetiva; sendo assim, cada um, a seu modo, sente sua dor. ${ }^{2}$ Acredita-se que o primeiro passo para avaliar a experiência álgica é confiar nas palavras e no comportamento do cliente, porém alguns cuidadores referem que às vezes o cliente não está com dor, só diz ter essa sensação para "chamar atenção" ou por "carência afetiva". Nesse momento, cabe à equipe de enfermagem rever as reais necessidades do cliente e cuidá-lo de maneira que essa dor seja amenizada, dando-lhe conforto, carinho, atenção e amor.

A dor é uma sensação desagradável que varia de intensidade e localização. Manifesta-se em cada indivíduo de maneira única, mesmo que ele passe por situações de injúrias semelhantes, pois a percepção da dor, e da reação a ela está na dependência da realidade de cada um, embora seja significativo que a maioria das pessoas identifiquem apenas sintomas orgânicos como dor, mas esta está entremeada por aspectos subjetivos, físicos e psíquicos, recebendo influência direta de fatores sociais, emocionais, culturais e espirituais. ${ }^{13}$

A melhor maneira de avaliar a dor é confiando nas palavras e no comportamento do cliente, acreditando que a dor existe. ${ }^{9}$ É ter a capacidade de perceber a experiência do outro, como ele vivencia; é estar atento para manter vivo o seu papel de cuidador, centrando a ação no ser humano, respeitando sua singularidade e seu modo próprio de existir. ${ }^{14} \mathrm{~A}$ assistência prestada ao cliente deve ser humanizada, atenciosa, respeitosa e justa, promovendo a interação e a comunicação entre os membros da equipe e o cliente, onde a dignidade pessoal do cliente seja valorizada. ${ }^{15}$

\section{Descrevendo os encontros com a equipe de enfermagem}

Após coletar os dados, foram realizadas quatro palestras com a equipe de enfermagem do CTI. Esses encontros fundamentaram-se em proporcionar uma reflexão e compreensão da necessidade da avaliação da dor, juntamente com os outros sinais vitais. Durante os encontros foi explicitado de maneira objetiva o conteúdo exposto na revisão de literatura. Na verdade, as palestras oportunizaram um diálogo entre pesquisadores e os participantes da pesquisa, onde se levantou dúvidas e contradições; contudo, chegou-se a um entendimento do assunto e percebeu-se a importância dessa temática, a fim de que o cliente possa ser percebido e valorizado na sua essência humana.

Cada indivíduo percebe e compreende o outro a partir da visão que tem de mundo. Por isso, é possível inferir que, para que o cuidado seja centrado na totalidade do ser, é mister que cuidadores detenham não só a competência técnica e o conhecimento científico, mas também a capacidade de compreender o ser humano como um ser relacional, que tem uma história de vida que deve ser respeitada e valorizada. ${ }^{14}$

Acredita-se que, após os encontros, a equipe de enfermagem do CTI sensibilizou-se com a temática e procurará considerar a queixa do cliente, compreendendo a subjetividade que a dor representa. Percebese que a enfermagem convive com esta problemática em seu cotidiano profissional e que existe o desejo de entender melhor a dor do outro, assim como saber avaliá-la e entendê-la como quinto sinal vital, fato que, sem dúvida, promoverá ações de cuidado humanizadas e de qualidade.

\section{CONSIDERAÇÕES FINAIS}

Ao término deste estudo, foi possível compreender que a equipe de enfermagem desta CTI apresentava um conhecimento insipiente quanto às formas de avaliar a dor. Desconhecia que esta é considerada o quinto sinal vital, uma vez que não tinha como prática 
avaliá-la sistematicamente. Portanto, a falta de conhecimento constitui-se em um desafio para o cuidar em enfermagem, sendo a educação em saúde um dos veículos capazes de promover instrução. Assim, a prática educativa é fundamental para o aperfeiçoamento da equipe de enfermagem, é a mola propulsora do conhecimento e do desenvolvimento das potencialidades e possibilidades do ser humano.

Nesse sentido, entende-se que a equipe deve receber orientações contínuas quanto à existência da norma que institui a dor como sendo o quinto sinal vital e estimulada a introduzir tal temática em sua prática profissional, a fim de que se possa prestar um cuidado mais qualificado ao cliente com dor, oportunizando um atendimento humanizado, onde a atenção é voltada às reais necessidades do cliente, valorizando e considerando sua queixa de dor. Assim, assegura-se que todos os clientes tenham acesso ao controle da dor.

Ao se propor realizar uma pesquisa, deve-se ter presente que ela é um veículo de mudanças sociais e comportamentais. Este estudo proporcionou à equipe de enfermagem do CTI momentos de reflexão, conhecimento, sensibilização e mudança comportamental, uma vez que a avaliação da dor foi instituída como o quinto sinal vital e hoje está presente nas ações de cuidado desta equipe.

O presente tema sensibilizou não somente os participantes da pesquisa, como também se refletiu na Coordenação de Enfermagem e Direção Hospitalar, que implantou o programa de avaliação da dor como quinto sinal vital em todas as unidades desta Instituição.

Foi possível perceber que a equipe, ao ser educada quanto à importância de avaliar a dor como quinto sinal vital, passou a ter um cuidado diferenciado e com maior qualidade. Portanto, acredita-se que a convergência cuidado-educação se constitui em um duplo desafio na prática da enfermagem, uma vez que para cuidar com qualidade é preciso manter-se atualizado e comprometido com a clientela. A educação em saúde, como um processo orientado para a capacitação do indivíduo, é capaz de modificar condutas que promovam ações de cuidado comprometidas com o ser humano e seu bem-estar. Acredita-se que as escolas médicas e de enfermagem, deveriam, urgentemente, implementar, em suas estruturas curriculares, disciplinas ou cursos com o propósito de ensinar e disseminar o uso de instrumentos e/ou escalas de avaliação e mensuração da dor, com uma perspectiva humanística.

\section{REFERÊNCIAS}

1 Carvalho MMMJ, organizadora. Dor: um estudo multidisciplinar. São Paulo: Summus; 1999.

2 Merskey H, Bogduk N, editors. Classification of chronic pain. Seattle: Internacional Association for the Study of Pain; 1994 [cited 2006 Abr 27]. Available from: http:// www.iasp-pain.org/terms-p.html

3 Booss J, Drake A, Kerns RD, Ryan B, Wasse L. Pain as the $5^{\text {th }}$ vital sign [toolkit on the internet]. Illinois: Joint Commission on Accreditation of Healthcare Organizations; 2000 [cited 2006 Abr 27]. Available from: http://www.va.gov/oaa/pocketcard/pain5thvitalsign/ Pain'Toolkit_Oct2000.doc

4 Bagatini A, Fuhrmeister AVA, Fortis EF, Nora F, Mendes F, Meyer I, et al. Anestesia: a vitória sobre a dor. Porto Alegre: SARGS; 2001.

5 Sakata RK, Hisatugo MKI, Aoki SS, Vlainich R, Issy AM. Avaliação da dor. In: Cavalcanti IL, Maddalena ML, editores. Dor. Rio de Janeiro: SAERJ; 2003. p.53-94.

6 Sousa FAEF, Hortense P. Mensuração da dor. In: Chaves LD, Leão ER, editores. Dor: $5^{\circ}$ sinal vital, reflexões e intervenções de enfermagem. Curitiba: Maio; 2004. p. $75-84$.

7 Ministério da Saúde (BR), Conselho Nacional de Saúde, Comitê Nacional de Ética em Pesquisa em Seres Humanos. Resolução No 196 de 10 de outubro de 1996: diretrizes e normas regulamentadoras pesquisa envolvendo seres humanos. Brasília (BR): O conselho; 1996.

8 Chaves LD. O processo de enfermagem no atendimento do indivíduo com dor. In: Chaves LD, Leão ER, editores. Dor: $5^{\circ}$ sinal vital: reflexões e intervenções de enfermagem. Curitiba: Maio; 2004. p.51-8.

9 Potter PA, Perry AG. A enfermagem no tratamento da dor. In: Potter PA, Perry AG. Grande tratado de enfermagem prática: clínica e prática hospitalar. São Paulo: Tempo; 2001. p.575-94.

10 Viana DL. Sensibilizando-se para cuidar: a experiência da enfermeira frente à avaliação da dor na criança [dissertação]. São Paulo (SP): USP/Programa de PósGraduação em Enfermagem; 2004.

11Clarke WC, Kashani A, Clark SB. A mensuração da dor. In: Kanner R. Segredos em clínica de dor. Porto Alegre: ArtMed; 1998. p. 41-50

12 Sousa FF. Dor: o quinto sinal vital. Rev. Latino-American. Enferm. 2002 Maio-Jun;10 (3): 446-7.

13 Paiva SS, Lindozo MAP. Dor em queimaduras. In: Chaves LD, Leão ER, editores. Dor: $5^{\circ}$ sinal vital: reflexões e intervenções de enfermagem. Curitiba: Maio; 2004. p.219-24. 
14 Celich KLS. Dimensões do processo de cuidar: a visão das enfermeiras. Rio de Janeiro: EPUB; 2004.
15 Chaves PL, Costa VT, Lunardi VL. A enfermagem frente aos direitos de pacientes hospitalizados. Texto Contexto Enferm. 2005 Jan-Mar; 14 (1):38-43. 